\title{
Potential of the incorporated collagen microspheres in alginate hydrogel as an engineered three- dimensional microenvironment to attenuate apoptosis in human pancreatic islets
}

\section{Maryam Kaviani}

Shiraz University of Medical Sciences https://orcid.org/0000-0002-3318-3363

\section{Somayeh Keshtkar}

Tehran University of Medical Sciences

Fatemeh Sabet Sarvestani

Shiraz University of Medical Sciences

Negar Azarpira ( $\nabla$ negarazarpira@gmail.com )

Ramin Yaghobi

Shiraz University of Medical Sciences

Mahdokht Hossein Aghdaei

Shiraz University of Medical Sciences

\section{Bita Geramizadeh}

Shiraz University of Medical Sciences

\section{Elaheh Esfandiari}

Shiraz University of Medical Sciences

\section{Alireza Shamsaeefar}

Shiraz University of Medical Sciences

\section{Saman Nikeghbalian}

Shiraz University of Medical Sciences

Ismail H. Al-Abdullah

City of Hope National Medical Center

Mohammad Hossein Karimi

Shiraz University of Medical Sciences

Nasrin Motazedian

Shiraz University of Medical Sciences

\section{Research}

Keywords: 
Posted Date: February 24th, 2020

DOl: https://doi.org/10.21203/rs.2.24328/v1

License: (c) (1) This work is licensed under a Creative Commons Attribution 4.0 International License. Read Full License

Version of Record: A version of this preprint was published at Acta Histochemica on October 1st, 2021. See the published version at https://doi.org/10.1016/j.acthis.2021.151775. 


\section{Abstract}

Background Tissue engineering is considered as a promising tool for remodeling the native cells microenvironment. In the present study, the effect of alginate hydrogel and collagen microspheres integrated with extracellular matrix components were evaluated in the decrement of apoptosis in human pancreatic islets. Methods For three dimensional culture, the islets were encapsulated in collagen microspheres, containing laminin and collagen IV and embedded in alginate scaffold for one week. After that the islets were examined in terms of viability, apoptosis, genes and proteins expression including BAX; BCL2; active caspase-3; and insulin. Moreover, the islets function was evaluated through the glucose-induced insulin and C-peptide secretion assay. In order to evaluate the scaffolds structure and the pancreatic islets morphology in three-dimensional microenvironments, scanning electron microscopy was done. Results Our findings showed that the designed hydrogel scaffolds significantly improved the islets viability the activated caspase-3 and TUNEL positive cells were clearly reduced. Conclusion The reduced pancreatic islet survival and function occurs, following the degradation of extracellular matrix and the stresses introduced into the cells during isolation and culture. The reconstruction of the destructed matrix with alginate hydrogels and collagen microspheres might be an effective step to promote the culture and transplantation of the islets.

\section{Background}

The isolation process of the human pancreatic islets from their niche leads to lose of biological and mechanical support of the cells. In this stressful situation, the apoptosis is activated in the islets; hence, eliminating this process becomes critical in order to reduce the number of tissues needed for pancreatic islet transplantation (1-4). Tissue engineering is an attractive strategy for remodeling extracellular matrix (ECM) and improving the culture and transplantation methods.

In order to design an efficient engineered tissue, attention to native tissue niche is necessary. In pancreas, the islets are surrounded by a capsule of collagen I, collagen IV, laminin, and fibronectin (5). Hydrogels are useful tools to encapsulate pancreatic islets (6). Cell encapsulation in hydrogels provides opportunities to eliminate immunological responses and ameliorate the cells viability and function during in vitro culture and transplantation. In many studies alginate was used for pancreatic islets encapsulation. Alginate is a biocompatible, inexpensive and available bioactive molecule (7). It was reported that this polysaccharide dose not interact with the pancreatic islets function $(8,9)$. In addition, the alginate is one of the few substances that can be processed in the form of hydrogel to encapsulate cells in physiological conditions (10). In this regard, Lee et al. evaluated the effect of alginate and alginate-collagen composite on pancreatic islets isolated from rat. They showed superiority of collagen-alginate composites on alginate. Moreover, xenotransplantation of these composites in BALB/c mice indicated that the cells had high viability and appropriate function to control the glucose level (11). On the other hand, previous findings showed that the application of ECM components including laminin and collagen IV facilitate the survival of mice pancreatic islets (12). 
In 2009, researchers used collagen-microspheres in alginate gel to improve the performance of glucose derived neurotrophic factor (GDNF)-secreting HEK293 cells (13). They found out that collagen microspheres in alginate gel increase the secretion level of GDNF over time. Therefore, this engineered structure was also potentiated to control the delivery of GDNF.

According to the importance of three dimensional (3D) microenvironment and ECM in the maintenance of cell integrity, viability, and function; cells microenvironment reconstruction can be a step forward in improving the cells implant outcome. This study was conducted to examine alginate hydrogel and collagen microspheres-containing the essential elements of ECM including collagen IV and lamininembedded in alginate hydrogel scaffolds for attenuating apoptosis in human pancreatic islets.

\section{Methods}

\section{Human pancreatic islets isolation and culture}

Human pancreases were harvested from Shiraz organ transplant center under ethical protocols approved by Shiraz University of Medical Sciences, and in accordance with the Code of Ethics from Declaration of Helsinki and its later amendments for experiments involving humans. The pancreas was digested in Richordi chamber containing several marbles and collagenase/neutral protease (Serva, Germany). After confirming the isolated islets by DTZ staining, purification step was done in a COBE 2991 cell processor using Biocoll (Biochrom, Germany) density gradient. The samples with more than $70 \%$ purity- the ratio of DTZ-stained part to the whole digested tissue- were plated in non-adherent cell culture flasks (Corning, Canada) in the presence of CMRL 1066 (Gibco, UK) supplemented with 1\% FBS (Gibco, UK), 1\% Lglutamine, $1 \%$ antibiotic-antimycotic (Sigma, Germany), and $6.25 \mu \mathrm{g} / \mathrm{ml}$ ITS (Sigma, Germany). The islets were cultured overnight in $5 \% \mathrm{CO}_{2}$ at $37^{\circ} \mathrm{C}(14-16)$.

\section{Scaffold preparation}

One thousand islet equivalents (IEQ) were assigned to each group, including control group without scaffold (Con.), Alginate hydrogel group (Alg.), collagen I microspheres incorporated in alginate hydrogel group (Coll. I \& Alg.), collagen I microspheres containing collagen IV incorporated in alginate hydrogel group (Coll. I, IV \& Alg.), collagen I microspheres containing laminin incorporated in alginate hydrogel group (Coll. I, Lam \& Alg.), and collagen I microspheres containing collagen IV and laminin incorporated in alginate hydrogel group (Coll. I, IV, Lam \& Alg.).

The pancreatic islets were encapsulated in the scaffolds according to a previous protocol with some modifications (13). In order to produce the microspheres, eight parts $2 \mathrm{mg} / \mathrm{ml}$ collagen I (Sigma, Germany) was mixed with one part 10X RPMI 1640. In the groups containing collagen IV or/and laminin, these ECM components were added with the final concentration of $1 \mathrm{mg} / \mathrm{ml}$. The $\mathrm{pH}$ of the mixture was adjusted to 7.4 with $1 \mathrm{M} \mathrm{NaOH}$, if needed. Then, the pancreatic islets of each group were mixed with the $100 \mu \mathrm{l}$ neutralized combination and dispensed as $2 \mu \mathrm{l}$ droplets onto a sterile $60 \mathrm{~mm}$ petri dish coated by UVirradiated parafilm. For gel formation to occur, the droplets were incubated at $37^{\circ} \mathrm{C}$ for $1 \mathrm{~h}$. After that the 
microspheres were cultured overnight in completed CMRL medium. The next day, the microspheres were transferred to a $15 \mathrm{ml}$ tube and settled for $15 \mathrm{~min}$ to separate the microspheres from the media.

In order to prepare alginate gel, 1000 IEQ pancreatic islets or 50 microspheres were suspended in $250 \mu$ l sodium alginate (Sigma, Germany) with a final concentration of $2 \%$. The mixture was then added to a $2 \mathrm{~cm}^{2}$ mold and incubated in $100 \mathrm{mM} \mathrm{CaCl}_{2}$ solution for $15 \mathrm{~min}$ at room temperature (Fig. 1). After that the gel was detached from the well and washed with normal saline. Finally, the scaffolds were placed in 6 $\mathrm{cm}$ culture plate in complete CMRL medium for one week.

\section{Inverted Microscope and Scanning Electron Microscope (SEM) analysis}

After one-week incubation in different groups, the morphological changes of the islets were evaluated under inverted microscope. To analyze the engineered structures and human pancreatic islets using SEM, we used a previous method with some modifications (11), the scaffolds were washed in PBS and fixed in $2.5 \%$ glutaraldehyde for $1 \mathrm{~h}$ (Sigma, Germany). The structures were dehydrated in serial dilutions of $25 \%$, $50 \%, 75 \%$, and $95 \%$ ethanol for 30 min each. The scaffolds were dried and analyzed under SEM. For observing the structures with the SEM (Tescan Vega3,) the samples were mounted on stubs and coated with gold.

\section{Viability of the pancreatic islets}

To examine the effect of scaffolds on pancreatic islets viability after one week, fluorescent dyes (Sigma, Germany) were applied (17). In this regard $5 \mathrm{mg} / \mathrm{ml}$ fluorescein diacetate (FDA) and $2 \mathrm{mg} / \mathrm{ml}$ propidium iodide (PI) were used to detect the live and dead cells, respectively. The stained islets were evaluated under fluorescent microscope (CKX53, Olympus, Japan). The viability rate was estimated by the ratio of FDA positive area to the whole of each islet.

\section{Gene expression in the pancreatic islets}

The expression of $B A X, B C L 2$, and $B A X / B C L 2$ ratio genes were evaluated by real time RT- PCR. RNA of the free and encapsulated islets were extracted by RNA-Sol isolation kit (Alphabio, Canada). Then, cDNA was synthesized using PrimeScript TM RT Reagent Kit (Takara, Japan). The primer design was done by NCBI tool Primer BLAST and GAPDH was considered as the housekeeping gene. The primer pairs were: GAPDH, F: GCTCATTTCCTGGTATGACAACG and R: CTCTCTTCCTCTTGTGCTCTTG; BCL2, F: GATGGGATCGTTGCCTTATGC and R: CAGTCTACTTCCTCTGTGATGTTGT; and BAX, F:

TTCTGACGGCAACTTCAACT and R: GGAGGAAGTCCAATG TCCAG. The amplification of the target was done by SYBR ${ }^{\circledR}$ Premix Ex TaqTM II (Takara, Japan), using Applied Biosystems StepOnePlus ${ }^{\text {TM }}$ Real-Time PCR system (ABI, USA) in 40 cycles. The fold changes of each gene was calculated by $2^{-\triangle \triangle C T}$.

\section{Protein expression in the pancreatic islets}

The assessment of BAX, BCL2, and active caspase-3 proteins expression was performed by immunocytochemistry method. The free and embedded islets in the scaffolds were fixed in $4 \%$ 
paraformaldehyde for $15 \mathrm{~min}$. The islets and the scaffolds were both embedded in low melting agar (Sigma, Germany) and paraffin (18). The cell blocks were sectioned at $5 \mu \mathrm{m}$ thickness.

Immunocytochemistry was performed using primary antibodies against BAX, BCL2, and active caspase-3 (abcam, USA). Incubation in primary antibody was done overnight. After incubation in secondary antibody (abcam, USA) for $1 \mathrm{~h}$, the positive cells were detected by 3,3'-Diaminobenzidine (DAB).

Counterstain was carried out with Hematoxylin staining. The samples were evaluated and the $\mathrm{H}$-score was calculated as follows: $\mathrm{H}$ score $=1 \times(\%$ light staining $)+2 \times(\%$ moderate staining $)+3 \times(\%$ strong staining) (19).

\section{Terminal deoxynucleotidyl transferase dUTP Nick-End Labeling (TUNEL) assay}

To determine the TUNEL positive cells, sections of cell blocks were assessed by Click-iT® Plus TUNEL Assay (life technology, France). In this regard, the sections were deparaffinized in xylene and serial dilutions of ethanol. The TdT reaction was conducted for $60 \mathrm{~min}$. After that Click-iT® Plus reaction was performed for $30 \mathrm{~min}$. DAPI (sigma, Germany) staining was used for counterstaining. The stained samples were analyzed under fluorescence microscope (CKX53, Olympus, Japan).

\section{Insulin and C-peptide stimulation indexes}

For insulin and C-peptide stimulation indexes assays, the pancreatic islets in control and scaffold groups were exposed to $2.8 \mathrm{mM}$ glucose in RPMI 1640 supplemented by $0.5 \%$ BSA. After $1 \mathrm{~h}$, the medium was collected. Next, the islets were incubated in $20 \mathrm{mM}$ glucose in RPMI 1640 supplemented by $0.5 \%$ BSA for $1 \mathrm{~h}$, and the medium was collected. The concentrations of Insulin and C-peptide in the collected culture media was determined via ELISA kits (Monobind, USA) and microplate spectrophotometer (BioTek Epoch $^{\mathrm{TM}}$, USA) at $450 \mathrm{~nm}$. Ratio of the secreted insulin or C-peptide concentration in $20 \mathrm{mM}$ to $2.8 \mathrm{mM}$ was calculated as stimulation index (20).

\section{Statistical analysis}

Statistical analysis was performed via Kruskal-Wallis and Post-hoc test in SPSS software and the charts were drawn in Graph Pad Prism 6 Program. The data are presented as mean \pm S.E. P $<0.05$ was considered to be statistically significant.

\section{Results}

\section{Morphological features of the human pancreatic islets were maintained in different scaffolds}

Subsequent to the culture of the islets in the presence and absence of hydrogels for one week, they were evaluated by inverted microscope. The pancreatic islets in different scaffolds preserved their native spherical structure. In the control group- free of scaffold- the integrity of the peripheral cells in the islets seemed to have somewhat distorted (Fig. 2). 
The evaluation of the islets and hydrogel structures by SEM revealed that the islets are visible as spherical structures with high integrity. However, the islets integrity was altered in the control group. SEM images demonstrated that the alginate hydrogel and microspheres produced a porous network. Yellow arrow in Fig.3B indicates that the alginate has a rough surface. Moreover, Fig. 3D, E, and F show that the islets have been implanted in the microspheres.

\section{The produced scaffolds maintained pancreatic islets viability}

The assessment of the islets using fluorescent dyes showed that in the absence of 3D microenvironment, the islets died. However, in the presence of alginate hydrogels and microspheres, the islets viability was significantly maintained $(P<0.001)$. There were no differences between scaffold groups for cellular viability (Fig. 4).

\section{The gene expression of BAX and BCL2 and the ratio of BAX/BCL2 were not altered in different scaffold groups}

The study of apoptotic (BAX) and anti-apoptotic (BCL2) genes expression and the BAX/BCL2 ratio showed that there was no significant difference between different groups of hydrogel scaffold $(P=0.285$ for $\mathrm{BAX}$ gene, $\mathrm{P}=0.152$ for $\mathrm{BCL} 2$ gene and $\mathrm{P}=0.744$ for $\mathrm{BAX} / \mathrm{BCL} 2$ ratio).

The results indicated that the expression of BAX in all groups containing microspheres was approximately lower than (Alg.) group. On the other hand, the expression of BCL2 had increased in all microsphere groups. In (Coll. I, IV, Lam \& Alg.) group the reduction of BAX gene expression and the increased of BCL2 gene expression was slightly higher than other groups. A comparison of BAX / BCL2 ratios showed that this ratio was lower in all microsphere groups especially in (Coll. I, IV, Lam \& Alg.) group. All in all, there were no significant differences in genes expression between the groups (Fig. 5).

\section{The presence of 3D microenvironment inhibited the activation of caspase-3 in human pancreatic islets}

Immunocytochemistry studies after one week of incubation showed that there were significant differences in the level of proteins expression (Fig. 6, 7, and 8). Evaluation of the BAX protein indicated that the expression of this apoptotic protein in the control group was more than the other groups. The reduction of this protein expression was significant in the hydrogel scaffold groups (Coll. I \& Alg.) ( $P$ $<0.001)$, (Coll. I, IV \& Alg.) $(P<0.001)$ and (Coll. I, IV, Lam \& Alg.) $(P=0.019)$.

In relation to the expression of anti-apoptotic protein, $\mathrm{BCL2}$, a slight decrease in the expression was observed in the hydrogel scaffold groups compared to the (Con.) group, but this decrease was not significant $(P=0.563)$.

Investigating the expression of active form of caspase-3 protein showed that the expression of this protein in the (Con.) group was significantly higher than all hydrogel scaffold groups $(P<0.001)$, and this decrease in expression was significant in all groups, except (Alg.) group. 


\section{The hydrogel scaffolds drastically reduced the TUNEL positive cells in human pancreatic islets}

The application of TUNEL technique on the pancreatic islets after one-week of incubation in different groups indicated that there is a significant difference between the number of TUNEL positive cells $(p<$ 0.001). A large number of cells in the (Con.) group were TUNEL positive while in the hydrogel scaffold groups ( $P<0.001$ for (Alg.), (Coll. I \& Alg.), (Coll. I, IV \& Alg.), and (Coll. I, Lam \& Alg.) groups and $P=0.032$ for (Coll. I, IV, Lam \& Al g.) group) they were negative or limited (Fig. 9).

\section{There was no statistically significant difference in insulin and C-peptide stimulation indexes between the different incubation conditions}

Subsequent to one-week incubation of the pancreatic islets, cells performance was investigated in the presence or absence of scaffolds. In this regard, after treating the cells with the concentrations of 2.8 and $20 \mathrm{mM}$ glucose, their insulin and C-peptide stimulation indexes were examined (Fig. 10A and B). The findings showed that there was no significant difference in insulin secretion $(P=0.791)$ and $C$-peptide $(P=$ 0.190 ) indexes between different groups.

\section{Discussion}

The mechanical and enzymatic digestion during pancreatic islet isolation leads to the destruction of ECM, resulting in loss of biological and mechanical protection. In this regard, tissue engineering is a promising way for restoring ECM by maintaining the structure and function of the cells. Recent studies have shown that the culture of human islets in 3D scaffolds improves the morphology, viability, and performance of the islets during pre-transplant stage.

In the present study, the effect of 3D hydrogel microenvironment with different compounds was used to evaluate viability and functionality of human pancreatic islets of Langerhans. For this purpose, the islets in different groups (Con.), (Alg.), (Coll. I \& Alg.), (Coll. I, IV \& Alg.), (Coll. I, Lam \& Alg.), and (Coll. I, IV, Lam \& Alg.) were incubated for one week and then examined for morphology, viability and functionality. In order to culture the islets in alginate hydrogel scaffolds, in four groups of; (Coll. I \& Alg.), (Coll. I, IV \& Alg.), (Coll. I, Lam \& Alg.), and (Coll. I, IV, Lam \& Alg.), first, the islets were encapsulated in the collagen I microspheres in the presence or absence of collagen IV and laminin, and finally placed in the alginate hydrogel platform.

The evaluation of islets structure with inverted optical microscopy showed the maintenance of the cells native morphology in the hydrogel scaffolds. The obtained results were confirmed by SEM. Our findings showed that spherical-3D morphology and integrated pancreatic islet structures were preserved in all hydrogel scaffolds groups. On the other hand, it seemed that structural integrity of the islets in the control group was relatively disturbed.

According to the Nomenclature Committee on Cell Death (NCCD), using fluorescent dyes as one of the criteria to confirm lack of membrane integrity and cell death, in this study, cell viability was examined with 
PI. It was clearly observed that the viability of the pancreatic islets in the course of one week in the control group was significantly reduced, while in hydrogel groups, it was completely preserved.

Previous studies showed that hydrogels, by simulating hydrophilic elements of the ECM, are desired tools for the encapsulation of the pancreatic islets. Recently, researchers have used various biomaterials and methods to achieve an optimal and efficient system for the reconstruction of the human pancreatic islets ECM. Cellular scaffolds design should be done in a way that they can mimic the inherent ECM, by maintaining cellular viability.

In this study, the main substrate of the hydrogels was alginate. Studies showed that the use of alginate polysaccharide has many advantages. Alginate is the most common biomaterial for islets encapsulation. Alginate capsule processing is possible in physiological conditions, including body temperature, physiological osmolarity and $\mathrm{pH}(21,22)$. Moreover, alginate is generally biocompatible and biodegradable. It has been shown that alginate is suitable for encapsulating cells and controlled delivery of bioactive molecules. In addition, this biomaterial can affect cell growth, metabolism and insulin production $(8,23,24)$. Investigation of the intrinsic microenvironment of human pancreatic islets showed that their ECM consists of collagen I, III, IV, V and VI, laminin and fibronectin $(25,26)$.

Zhang et al. studied the survival of pancreatic islets in a collagen matrix of type I for 7 days. They found that cellular viability was significantly higher than that of the control group. In addition, the islets enclosed in the collagen matrix had a much lower number of active caspase-expressing cells than the control group (27). Lee et al. also made a comparison between the rat pancreatic islets cultured in the alginate and the alginate-collagen type I composite, and observed that the used composite remarkably improved the cells viability (11). On the application of ECM factors, researchers examined the survival rate of mice Islets in silk hydrogel containing laminin and collagen IV. This study showed that Silk alone can help to increase islets survival, and adding laminin and IV collagen cannot promote this hydrogel (12). In a previous study, perfluorodecalin-enriched fibrin matrix led to the reduction of caspase-3 activation in cultured human pancreatic islets. This structure provided a favorable chemical and physical environment for these cells, but the study was only performed within 24 hours (28). In general, studies on the evaluation of apoptosis following the use of 3D scaffolds in human pancreatic islets are very limited.

In the present study, we observed that in all groups with hydrogel scaffolds the viability of human pancreatic islets was significantly higher than the control group, and the presence of collagen and laminin did not have any superiority over pure alginate hydrogel. In total, all scaffolds designed in this study were able to fully maintain the viability of the islets (Fig. 11).

Considering the importance of cellular life in the prognosis of pancreatic islet transplantation, it seems essential to investigate the cause of islets' death in order to prevent this destructive process. In this regard, the present study focused on the internal pathway of apoptosis - as the main cause of pancreatic islets death. Therefore, the expression of genes and proteins involved in the process of apoptosis and the number of positive TUNEL cells was evaluated. 
Although the analysis of $B A X$ and BCL2 genes expression and the BAX/BCL2 ratio reveled that there was no significant difference between different groups of scaffolds, the increased of BCL2 gene and the decreased of $B A X$ gene and $B A X / B C L 2$ ratio was visible. On the other hand, the evaluation of $B A X, B C L 2$ and active caspase-3 proteins indicated significant differences between hydrogel scaffold groups and non-scaffold group. BAX expression decreased in all scaffold groups, but contrary to the expectation, the expression of BCL2 showed a non-significant reduction. Furthermore, we found out that the expression of active caspase-3 was reduced in all hydrogel scaffold groups. This reduction was significant in the surrounded microspheres by alginate hydrogel. The contradiction between significant differences at the level of genes and proteins expression might have been due to the effect of scaffolds on posttranslational proteins modifications.

In order to further clarify the cell death pathway, after the evaluation of active caspase-3 protein expression, the number of positive TUNEL cells were measured. In the control group, a large number of cells were TUNEL positive, as the high expression of active caspase-3 and enormous cell death was evident. However, the findings showed that although caspase-3 expression was slightly expressed in 3Dscaffold groups, very limited number of cells were TUNEL positive. This discrepancy may suggests that caspase- 3 activation is not necessarily indicative of apoptosis (29). On the other hand, it seems that there is a threshold for the destructive activity of active caspase-3. Geske et al. showed that apoptotic cells induced by $p 53$ could be released from apoptosis during the removal of apoptotic stimulus. Their study suggested that DNA repair could be activated in this process and in some cases results in a return from the cell death pathway (30). All in all, studies suggest that the mechanisms involved in apoptosis are complex and a cascade of molecular events guides intrinsic and extrinsic apoptosis. Previous evidences are based on the fact that these two pathways are interconnected and the molecules involved in one path can affect the other (31).

The performance of pancreatic islets is an indicator of the islets transplantation prognosis. In this study, the evaluation of insulin and C-peptide secretion indexes suggested that hydrogel scaffolds do not have an adverse effect on the secretory function of these islets. Although the differences were not statistically significant, the improvement in secretion indices was visible in some groups. The islets of the (Alg.) group showed a higher level of both indices. Thus, the alginate hydrogel microenvironment might provide a favorable 3D-microenvironment for these cells.

With respect to the application of collagen microspheres, Wang et al. study suggested that collagen microspheres, compared with single-layer cultures, increases the production and secretion of GDNF from HEK293 cells (13). Subsequently, Lee et al. embedded these cells in collagen microspheres, incorporated in alginate hydrogel and compared GDNF secretion in these conditions with cells embedded in collagen I and alginate composites (13). They found that in the composite, stable release of GDNF occurred throughout the entire culture period, and its release level was controlled by various concentrations of alginate. On the other hand, due to proliferation of HEK293 cells in collagen microspheres enclosed in alginate scaffolds, GDNF release was increased steadily. 
In the present study, there was no significant differences in the index of insulin and C-peptide secretion between the groups containing microspheres and the control group. Overall, the 3D scaffolds designed in this study had no adverse effect on the activity of pancreatic islands.

\section{Conclusion}

Due to limited survival and efficiency of the pancreatic islets, following the destruction of ECM during the isolation process, rebuilding appropriate matrix can be an effective step to promote their cultivation and transplantation. Based on the obtained results and the apparent effect of the designed scaffolds on the viability of human pancreatic islets, there is a possibility to consider them as cell carriers in regenerative medicine.

\section{Abbreviations}

Extracellular Matrix: ECM

Glucose Derived Neurotrophic Factor: GDNF

Islet Equivalents (IEQ)

Control group without scaffold: Con.

Alginate hydrogel group: Alg.

Collagen I microspheres incorporated in alginate hydrogel group: Coll. I \& Alg.

Collagen I microspheres containing collagen IV incorporated in alginate hydrogel group: Coll. I, IV \& Alg.

Collagen I microspheres containing laminin incorporated in alginate hydrogel group: Coll. I, Lam \& Alg.

Collagen I microspheres containing collagen IV and laminin incorporated in alginate hydrogel group:

(Coll. I, IV, Lam \& Alg.).

Scanning Electron Microscope: SEM

Fluorescein Diacetate: FDA

Propidium lodide: PI

Terminal Deoxynucleotidyl Transferase Dutp Nick-End Labeling: TUNEL

Nomenclature Committee on Cell Death: NCCD

\section{Declarations}


- Ethics approval and consent to participate

Human pancreases were harvested from Shiraz organ transplant center under ethical protocols approved by Shiraz University of Medical Sciences, and in accordance with the Code of Ethics from Declaration of Helsinki and its later amendments for experiments involving humans

- Consent for publication

“Not applicable"

- Availability of data and materials

The datasets used and/or analysed during the current study are available from the corresponding author on reasonable request

- Competing interests

"The authors declare that they have no competing interests"

- Funding

financial support by Transplant Research Center and Deputy of Research and Technology, Shiraz University of Medical Sciences, Shiraz, Iran (Grant number: 94.521449) and National Institute for Medical Research Development (NIMAD) (Grant number: 943731).

- Authors' contributions

M.K.: Design of the work, acquisition, analysis, and interpretation of the data; drafting the work.

S. K.: Performed the pancreatic islets isolation procedure, data gathering, and the article revision.

F. S. S.: Performed the pancreatic islets isolation procedure, and the article revision.

N.A.: Design of the work, coordinate the study, interpretation of the data, and the article revision.

M.HA.: Performed the pancreatic islets isolation procedure, and the article revision.

B.G.: Interpretation of the data, and the article revision.

MH.K. and R.Y.: Interpretation of the data, and the article revision.

E.E.: Performed the pancreatic islets isolation procedure, and the article revision.

A.SH. and N.M.: Coordinated the study, interpretation of the data, and the article revision.

S.N.: Interpretation of the data, and the article revision. 
IH.AA.: Interpretation of the data, and the article revision.

All authors approved the manuscript for the submission. They agree to be accountable for all aspects of the work.

- Acknowledgements

The authors gratefully thank the financial support by Transplant Research Center and Deputy of Research and Technology, Shiraz University of Medical Sciences, Shiraz, Iran (Grant number: 94.521449) and National Institute for Medical Research Development (NIMAD) (Grant number: 943731). The authors wish to thank Mr. H. Argasi at the Research Consultation Center (RCC) of Shiraz University of Medical Sciences for his invaluable assistance in editing this manuscript.

\section{References}

1. Keshtkar S, Kaviani M, Jabbarpour Z, Geramizadeh B, Motevaseli E, Nikeghbalian S, et al. Protective effect of nobiletin on isolated human islets survival and function against hypoxia and oxidative stress-induced apoptosis. Scientific reports. 2019;9(1):1-13.

2. Kaviani M, Keshtkar S, Azarpira N, Aghdaei MH, Geramizadeh B, Karimi MH, et al. Cytoprotective effects of ginsenoside Rd on apoptosis-associated cell death in the isolated human pancreatic islets. EXCLI Journal. 2019;18:666-76.

3. Kaviani M, Keshtkar S, Azarpira N, Aghdaei MH, Geramizadeh B, Karimi MH, et al. Amelioration of the apoptosis-mediated death in isolated human pancreatic islets by minocycline. Eur $\mathrm{J}$ Pharmacol. 2019;858:172518.

4. Kaviani M, Keshtkar S, Azarpira N, Aghdaei MH, Geramizadeh B, Karimi MH, et al. Cytoprotective effects of olesoxime on isolated human pancreatic islets in order to attenuate apoptotic pathway. Biomed Pharmacother. 2019;112:108674.

5. Wang R, Rosenberg L. Maintenance of beta-cell function and survival following islet isolation requires re-establishment of the islet-matrix relationship. J Endocrinol. 1999;163(2):181-90.

6. Liao SW, Rawson J, Omori K, Ishiyama K, Mozhdehi D, Oancea AR, et al. Maintaining functional islets through encapsulation in an injectable saccharide-peptide hydrogel. Biomaterials. 2013;34(16):3984-91.

7. Orive G, Ponce S, Hernandez R, Gascon A, Igartua M, Pedraz J. Biocompatibility of microcapsules for cell immobilization elaborated with different type of alginates. Biomaterials. 2002;23(18):3825-31.

8. Lamb M, Storrs R, Li S, Liang O, Laugenour K, Dorian R, et al., editors. Function and viability of human islets encapsulated in alginate sheets: in vitro and in vivo culture. Transplant Proc; 2011: Elsevier.

9. Vaithilingam V, Quayum N, Joglekar MV, Jensen J, Hardikar AA, Oberholzer J, et al. Effect of alginate encapsulation on the cellular transcriptome of human islets. Biomaterials. 2011;32(33):8416-25. 
10. Kong HJ, Smith MK, Mooney DJ. Designing alginate hydrogels to maintain viability of immobilized cells. Biomaterials. 2003;24(22):4023-9.

11. Lee BR, Hwang JW, Choi YY, Wong SF, Hwang YH, Lee DY, et al. In situ formation and collagenalginate composite encapsulation of pancreatic islet spheroids. Biomaterials. 2012;33(3):837-45.

12. Davis NE, Beenken-Rothkopf LN, Mirsoian A, Kojic N, Kaplan DL, Barron AE, et al. Enhanced function of pancreatic islets co-encapsulated with ECM proteins and mesenchymal stromal cells in a silk hydrogel. Biomaterials. 2012;33(28):6691-7.

13. Lee M, Lo A, Cheung P, Wong D, Chan B. Drug carrier systems based on collagen-alginate composite structures for improving the performance of GDNF-secreting HEK293 cells. Biomaterials. 2009;30(6):1214-21.

14. Azarpira N, Aghdai MH, Nikeghbalian S, Geramizadeh B, Darai M, Esfandiari E, et al. Human islet cell isolation: the initial step in an islet transplanting program in Shiraz, Southern Iran. Experimental and clinical transplantation: official journal of the Middle East Society for Organ Transplantation. 2014;12(2):139-42.

15. Shapiro AJ, Ricordi C, Hering BJ, Auchincloss H, Lindblad R, Robertson RP, et al. International trial of the Edmonton protocol for islet transplantation. New Engl J Med. 2006;355(13):1318-30.

16. Bentsi-Barnes K, Doyle ME, Abad D, Kandeel F, Al-Abdullah I. Detailed protocol for evaluation of dynamic perifusion of human islets to assess $\beta$-cell function. Islets. 2011;3(5):284-90.

17. Ramírez-Domínguez $M$, Castaño L. Filtration is a time-efficient option to Histopaque, providing goodquality islets in mouse islet isolation. Cytotechnology. 2015;67(2):199-206.

18. da Cunha CB, Klumpers DD, Li WA, Koshy ST, Weaver JC, Chaudhuri O, et al. Influence of the stiffness of three-dimensional alginate/collagen-I interpenetrating networks on fibroblast biology. Biomaterials. 2014;35(32):8927-36.

19. Detre S, Jotti GS, Dowsett M. A" quickscore" method for immunohistochemical semiquantitation: validation for oestrogen receptor in breast carcinomas. J Clin Pathol. 1995;48(9):876-8.

20. Labriola L, Montor WR, Krogh K, Lojudice FH, Genzini T, Goldberg AC, et al. Beneficial effects of prolactin and laminin on human pancreatic islet-cell cultures. Mol Cell Endocrinol. 2007;263(12):120-33.

21. Pawar SN, Edgar KJ. Alginate derivatization: a review of chemistry, properties and applications. Biomaterials. 2012;33(11):3279-305.

22. de Vos P, Faas MM, Strand B, Calafiore R. Alginate-based microcapsules for immunoisolation of pancreatic islets. Biomaterials. 2006;27(32):5603-17.

23. Qi M, Strand BL, Mørch Y, Lacík I, Wang Y, Salehi P, et al. Encapsulation of human islets in novel inhomogeneous alginate-Ca2+/Ba2+ microbeads: in vitro and in vivo function. Artificial Cells, Blood Substitutes, and Biotechnology. 2008;36(5):403-20.

24. Köllmer M, Appel AA, Somo SI, Brey EM. Long-Term Function of Alginate-Encapsulated Islets. Tissue Engineering Part B: Reviews. 2015. 
25. Van Deijnen J, Van Suylichem P, Wolters G, Van Schilfgaarde R. Distribution of collagens type I, type III and type V in the pancreas of rat, dog, pig and man. Cell Tissue Res. 1994;277(1):115-21.

26. Stendahl JC, Kaufman DB, Stupp SI. Extracellular matrix in pancreatic islets: relevance to scaffold design and transplantation. Cell Transplant. 2009;18(1):1.

27. Zhang Y, Jalili RB, Warnock GL, Ao Z, Marzban L, Ghahary A. Three-dimensional scaffolds reduce islet amyloid formation and enhance survival and function of cultured human islets. The American journal of pathology. 2012;181(4):1296-305.

28. Maillard E, Juszczak MT, Clark A, Hughes SJ, Gray DR, Johnson PR. Perfluorodecalin-enriched fibrin matrix for human islet culture. Biomaterials. 2011;32(35):9282-9.

29. Elmore S. Apoptosis: a review of programmed cell death. Toxicol Pathol. 2007;35(4):495-516.

30. Geske F, Lieberman R, Strange R, Gerschenson L. Early stages of p53-induced apoptosis are reversible. Cell Death Differ. 2001;8(2):182.

31. Igney FH, Krammer PH. Death and anti-death: tumour resistance to apoptosis. Nature Reviews Cancer. 2002;2(4):277.

\section{Figures}

A $\quad$ B
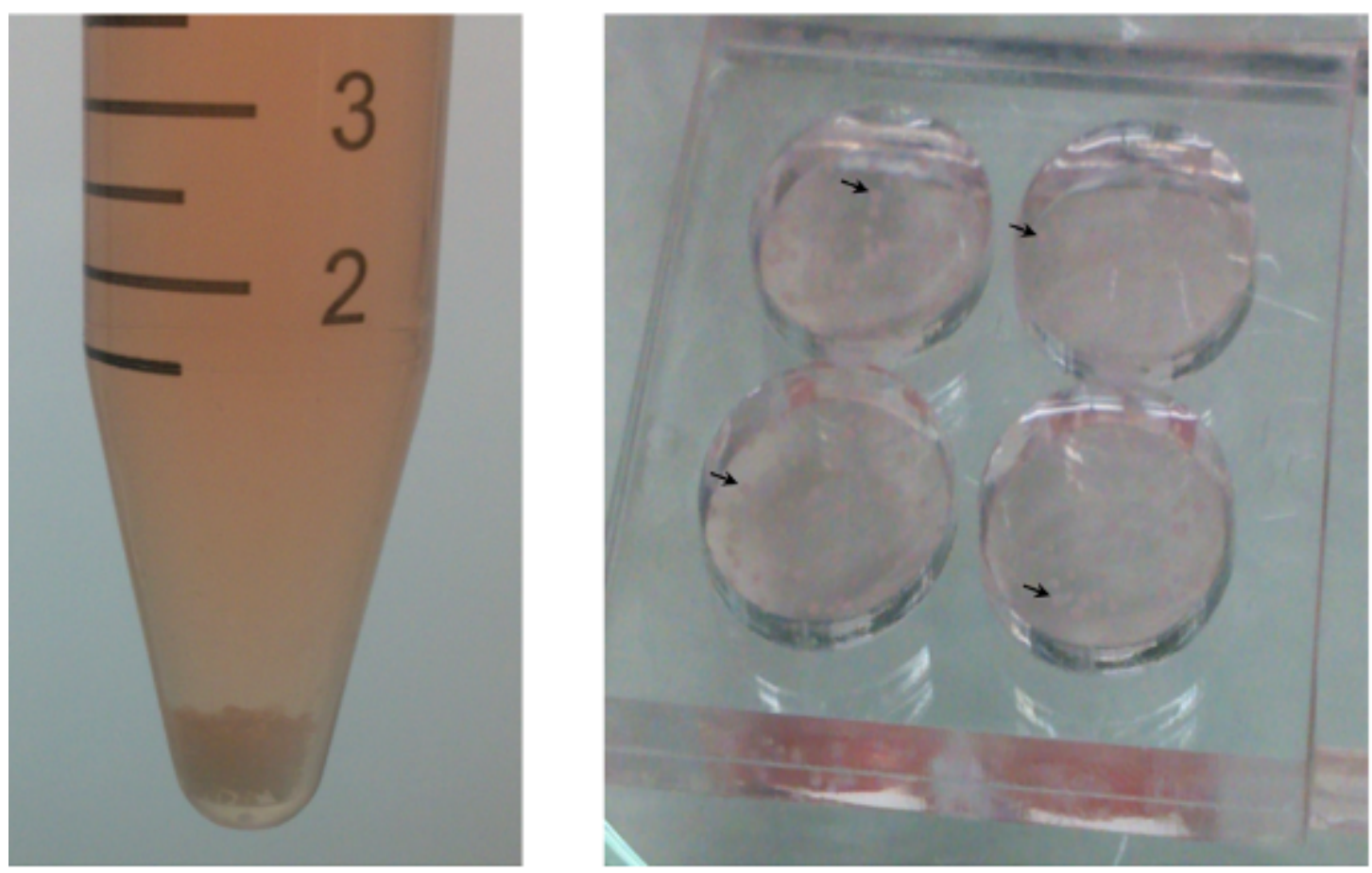

Figure 1

Scaffold preparation. Sedimentation of the microspheres after 15 minutes (A) and molding of the sodium alginate solution (B). The arrows indicate the microspheres in alginate gel. 


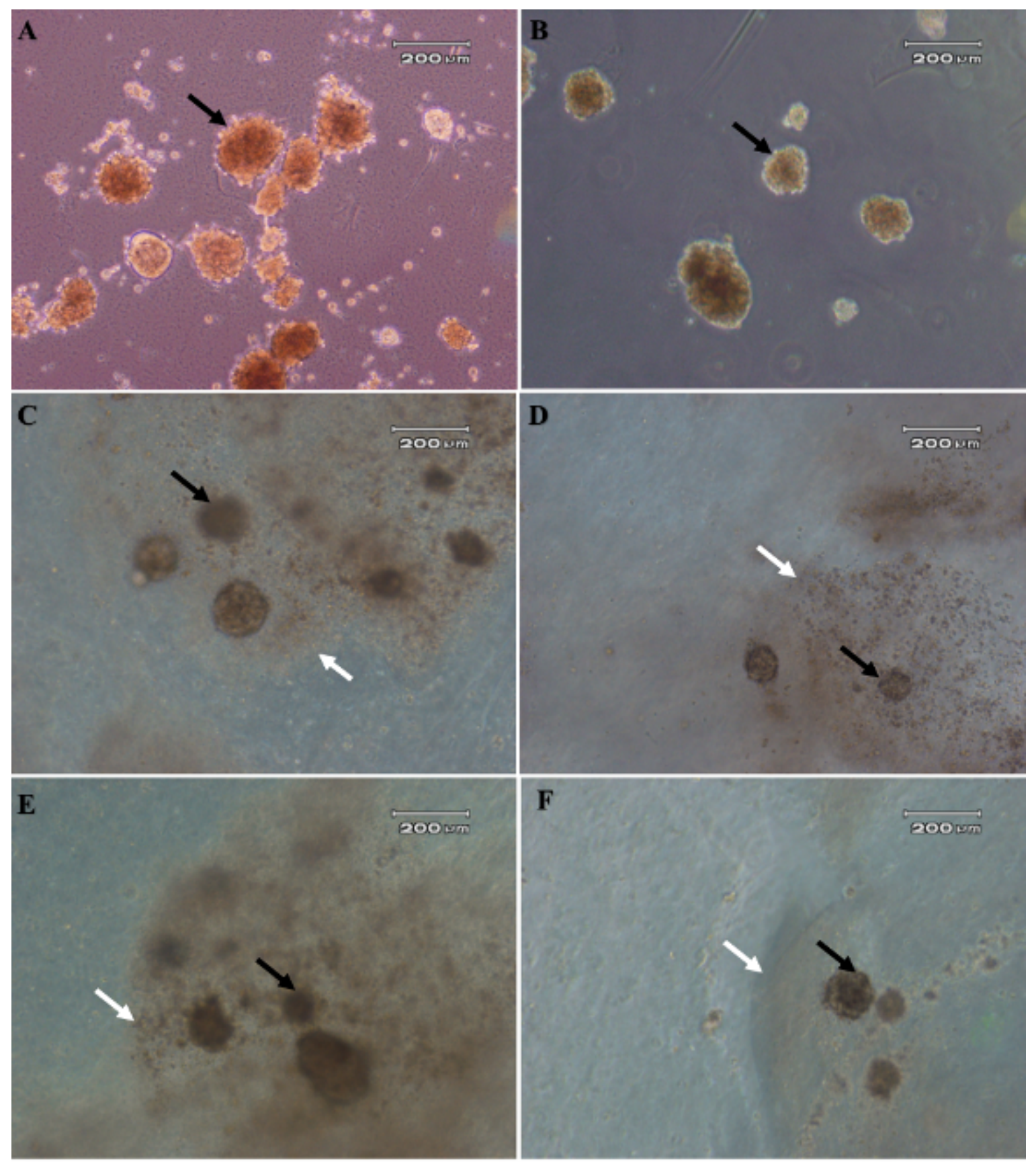

\section{Figure 2}

Microscopic view of the pancreatic islets in different groups. The islets maintained their spherical morphology in different hydrogel groups. The black arrows indicate the pancreatic islets and the white arrows show the microspheres. A: Control group, B: Alginate hydrogel group, C: Collagen I microspheres incorporated in alginate hydrogel group, D: Collagen I microspheres containing collagen IV incorporated in alginate hydrogel group, E: Collagen I microspheres containing laminin incorporated in alginate hydrogel group, and F: Collagen I microspheres containing collagen IV and laminin incorporated in alginate hydrogel group. 

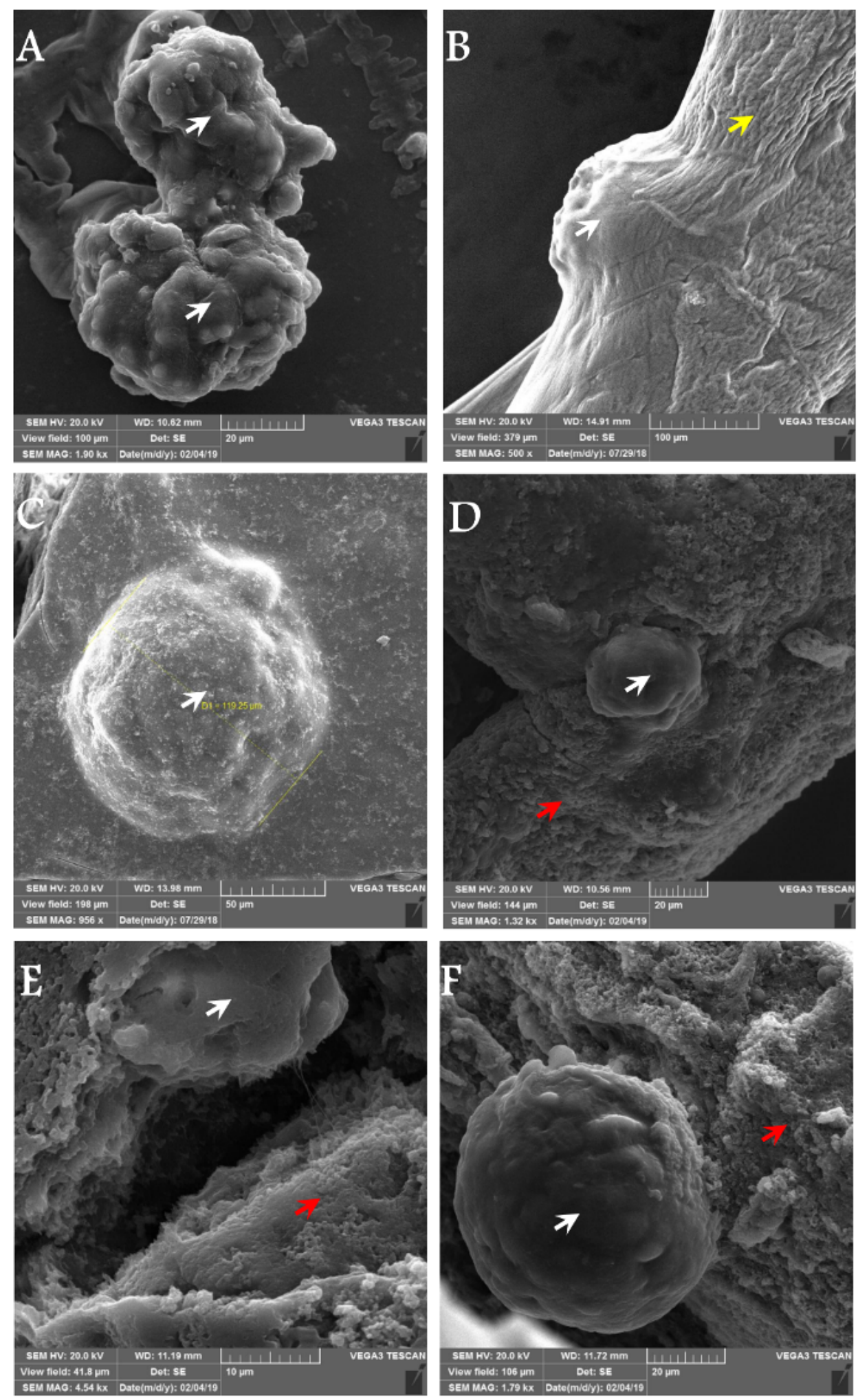

\section{Figure 3}

SEM view of the islets in different groups. The islets maintained their spherical morphology in different hydrogel groups. The yellow arrow indicates the rough surface of alginate hydrogel. The white arrows show the pancreatic islets and the red arrows depict the microspheres structures. A: Control group, B: Alginate hydrogel group, C: Collagen I microspheres incorporated in alginate hydrogel group, D: Collagen I microspheres containing collagen IV incorporated in alginate hydrogel group, E: Collagen I microspheres 
containing laminin incorporated in alginate hydrogel group, and F: Collagen I microspheres containing collagen IV and laminin incorporated in alginate hydrogel group.

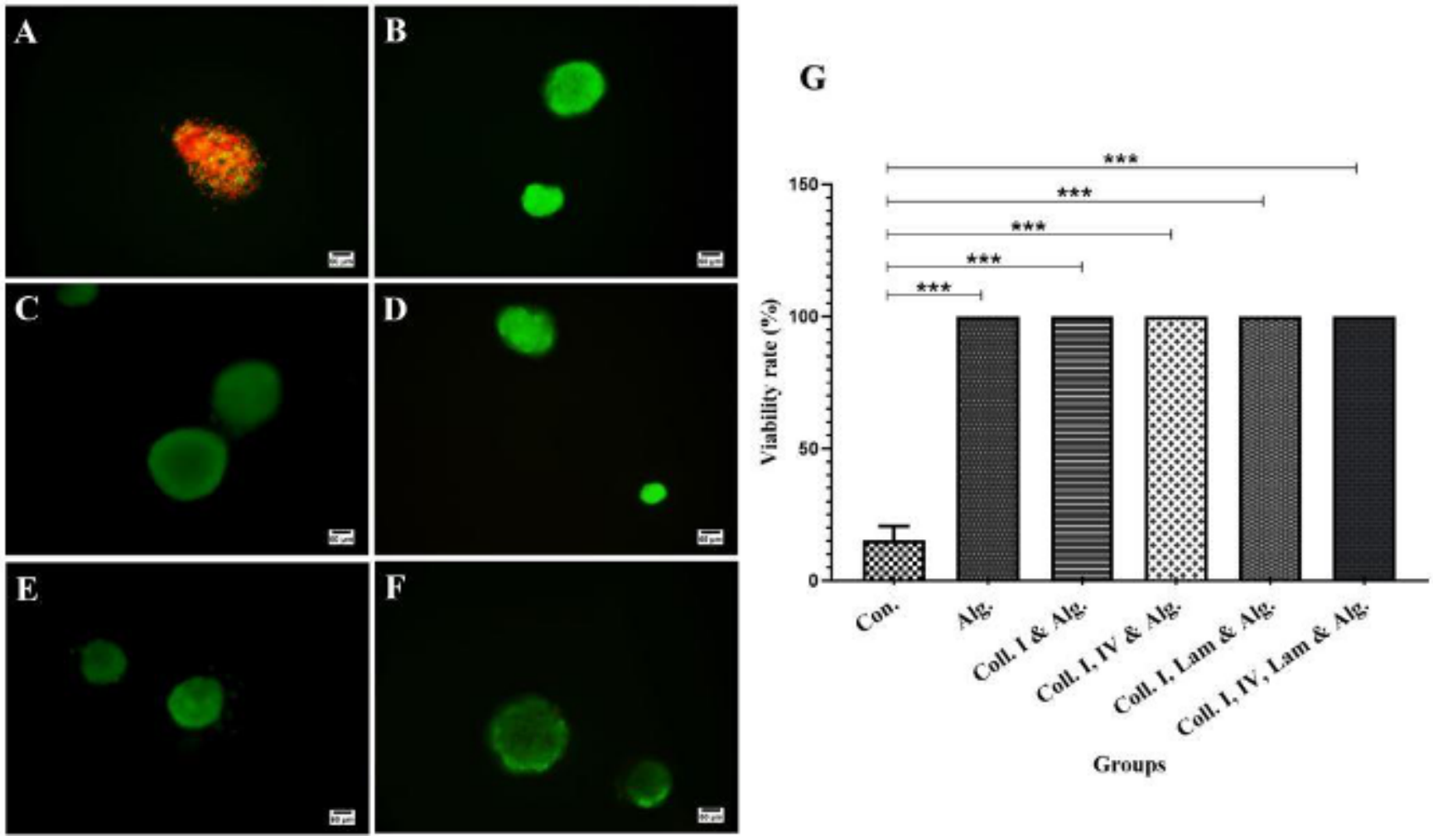

\section{Figure 4}

The viability of human pancreatic islets in different groups. Live and dead cells were stained by FDA and $\mathrm{PI}$, respectively. Fluorescent microscopy images were taken separately by the appropriate filter and then were mixed with Image $\mathrm{J}$ software $(\mathrm{A}-\mathrm{F})$. As shown in the illustrations, in the control group (A), a large number of cells died, but in the presence of hydrogel scaffolds in alginate hydrogel (B), collagen I microspheres incorporated in alginate hydrogel (C), collagen I microspheres containing collagen IV incorporated in alginate hydrogel (D), collagen I microspheres containing laminin incorporated in alginate hydrogel (E), and collagen I microspheres containing collagen IV and laminin incorporated in alginate hydrogel $(F)$ the cells survived. The viability rate histogram shows significant differences between the islets viability in 3D microenvironments compared to the control group (G). Data are reported as Mean \pm SEM. ***: P<0.001). A: Control group (Con.), B: Alginate hydrogel group (Alg.), C: Collagen I microspheres incorporated in alginate hydrogel group (Coll. I \& Alg.), D: Collagen I microspheres containing collagen IV incorporated in alginate hydrogel group (Coll. I, IV \& Alg.), E: Collagen I microspheres containing laminin incorporated in alginate hydrogel group (Coll. l, Lam \& Alg.), and F: Collagen I microspheres containing collagen IV and laminin incorporated in alginate hydrogel group (Coll. I, IV, Lam \& Alg.). 


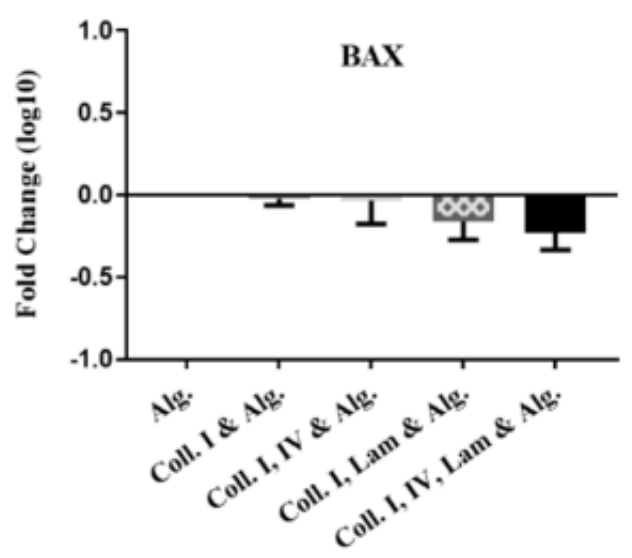

Groups

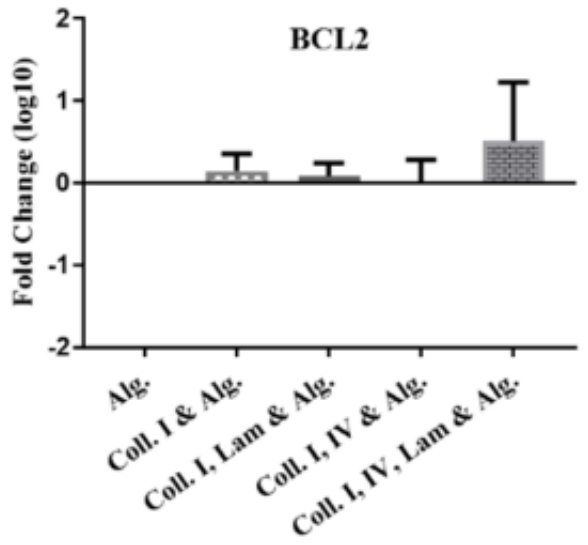

Groups

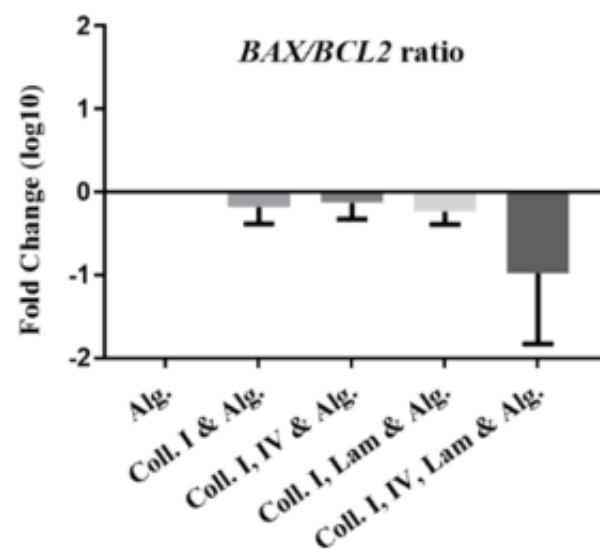

Groups

Figure 5

The expression of apoptotic (BAX) and anti-apoptotic (BCL2) genes and the ratio of them between different scaffold groups. (Alg.): Alginate hydrogel group, (Coll. I \& Alg.): Collagen I microspheres incorporated in alginate hydrogel group, (Coll. I, IV \& Alg.): Collagen I microspheres containing collagen IV incorporated in alginate hydrogel group, (Coll. I, Lam \& Alg.): Collagen I microspheres containing laminin incorporated in alginate hydrogel group, and (Coll. I, IV, Lam \& Alg.): Collagen I microspheres containing collagen IV and laminin incorporated in alginate hydrogel group.

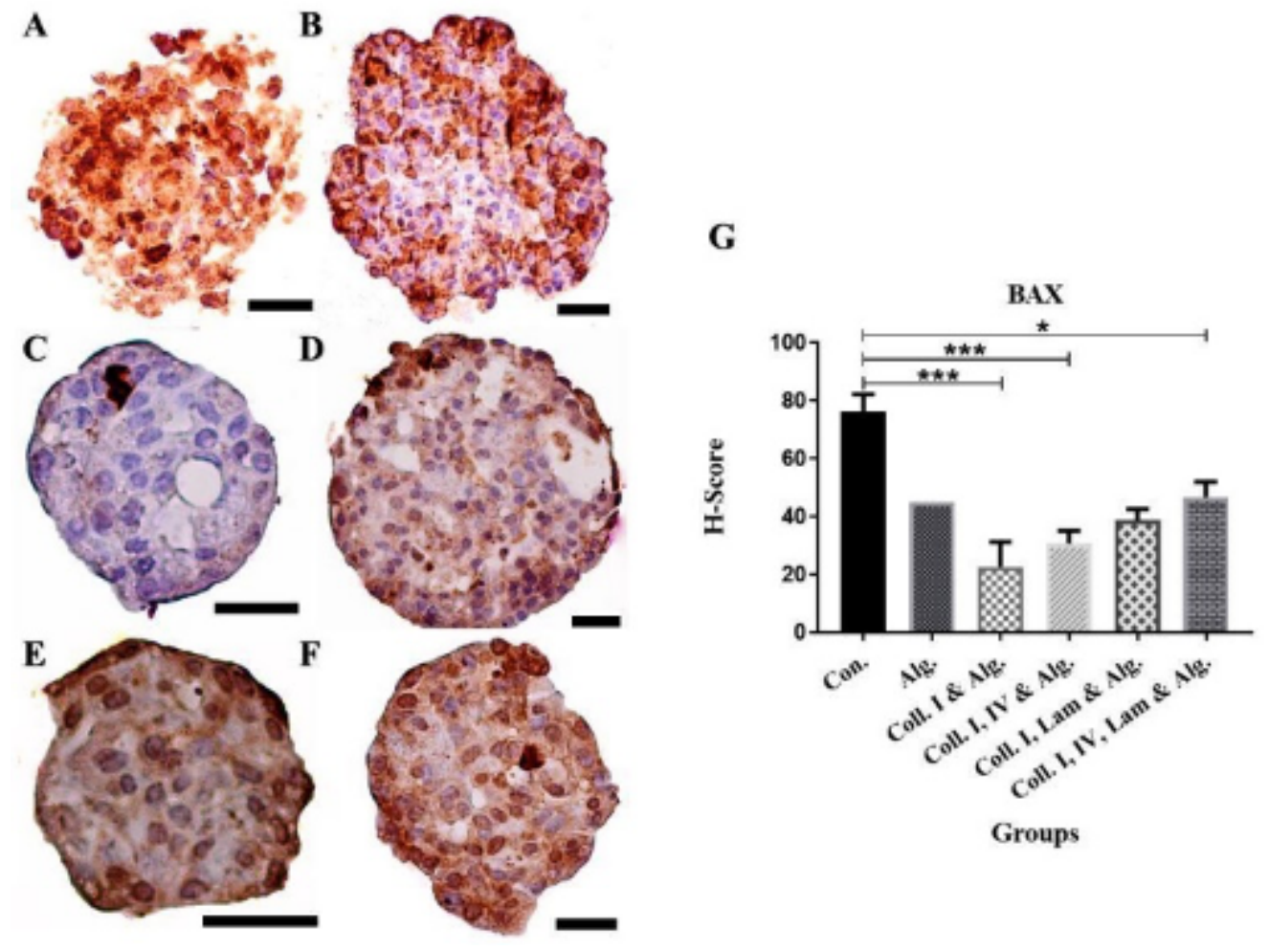

Figure 6

Immunocytochemistry analysis of BAX protein expression in different groups. The brown spots are the positive areas and the nuclei are visible in blue (A-F). The histogram shows the H-score in each group (G). Data are reported as Mean \pm SEM. (Measurement index of images $=20 \mu \mathrm{m}$. *: $P<0.05$ and ***: $P<0.001$ ). 
A: Control group (Con.), B: Alginate hydrogel group (Alg.), C: Collagen I microspheres incorporated in alginate hydrogel group (Coll. I \& Alg.), D: Collagen I microspheres containing collagen IV incorporated in alginate hydrogel group (Coll. I, IV \& Alg.), E: Collagen I microspheres containing laminin incorporated in alginate hydrogel group (Coll. I, Lam \& Alg.), and F: Collagen I microspheres containing collagen IV and laminin incorporated in alginate hydrogel group (Coll. I, IV, Lam \& Alg.).

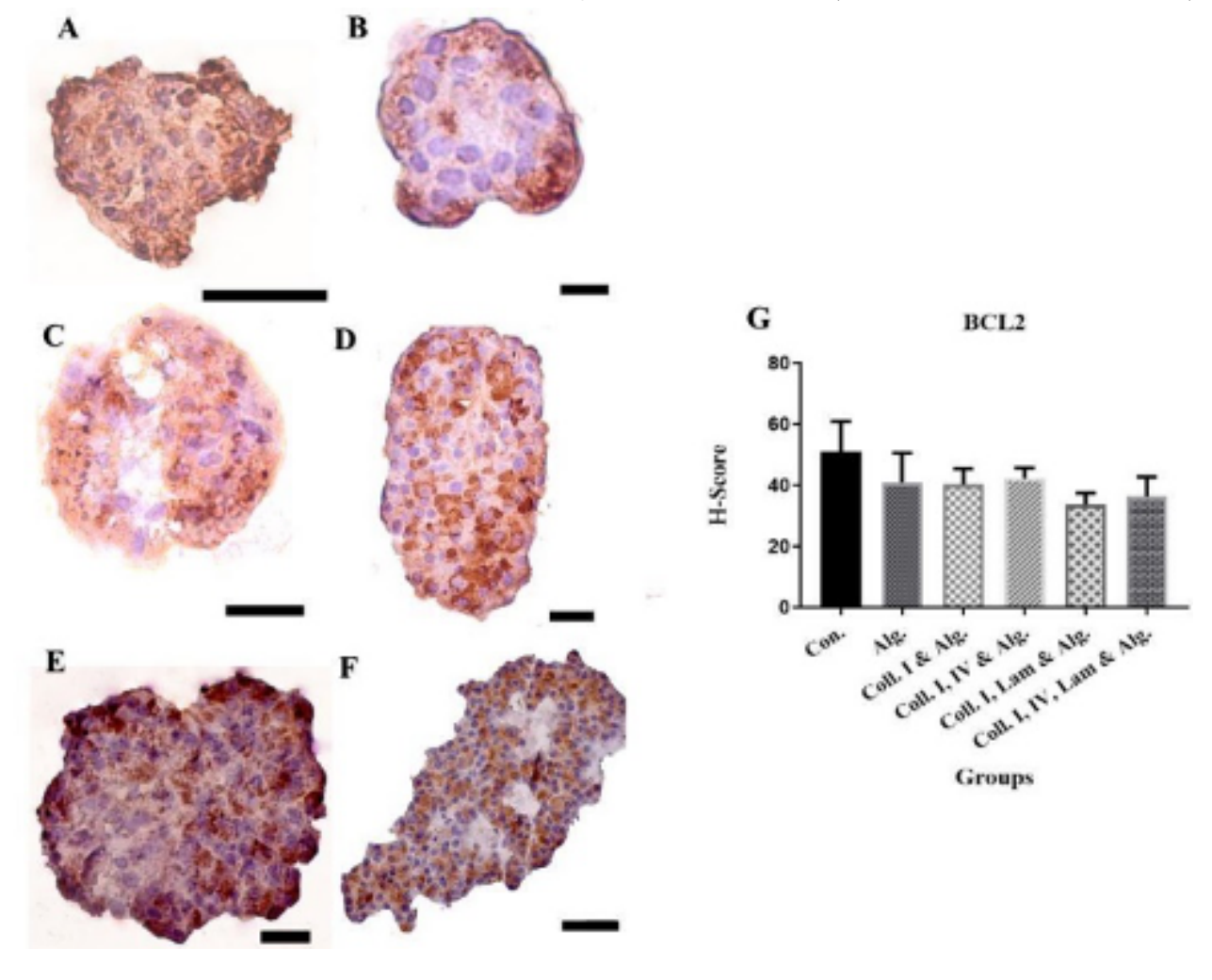

Figure 7

Immunocytochemistry analysis of BCL2 protein expression in different groups. The brown spots are the positive areas and the nuclei are visible in blue (A-F). The histogram shows the $\mathrm{H}$-score in each group (G). Data are reported as Mean \pm SEM. (Measurement index of images $=20 \mu \mathrm{m}) \mathrm{A}$ : Control group (Con.), B: Alginate hydrogel group (Alg.), C: Collagen I microspheres incorporated in alginate hydrogel group (Coll. I \& Alg.), D: Collagen I microspheres containing collagen IV incorporated in alginate hydrogel group (Coll. I, IV \& Alg.), E: Collagen I microspheres containing laminin incorporated in alginate hydrogel group (Coll. I, Lam \& Alg.), and F: Collagen I microspheres containing collagen IV and laminin incorporated in alginate hydrogel group (Coll. I, IV, Lam \& Alg.). 


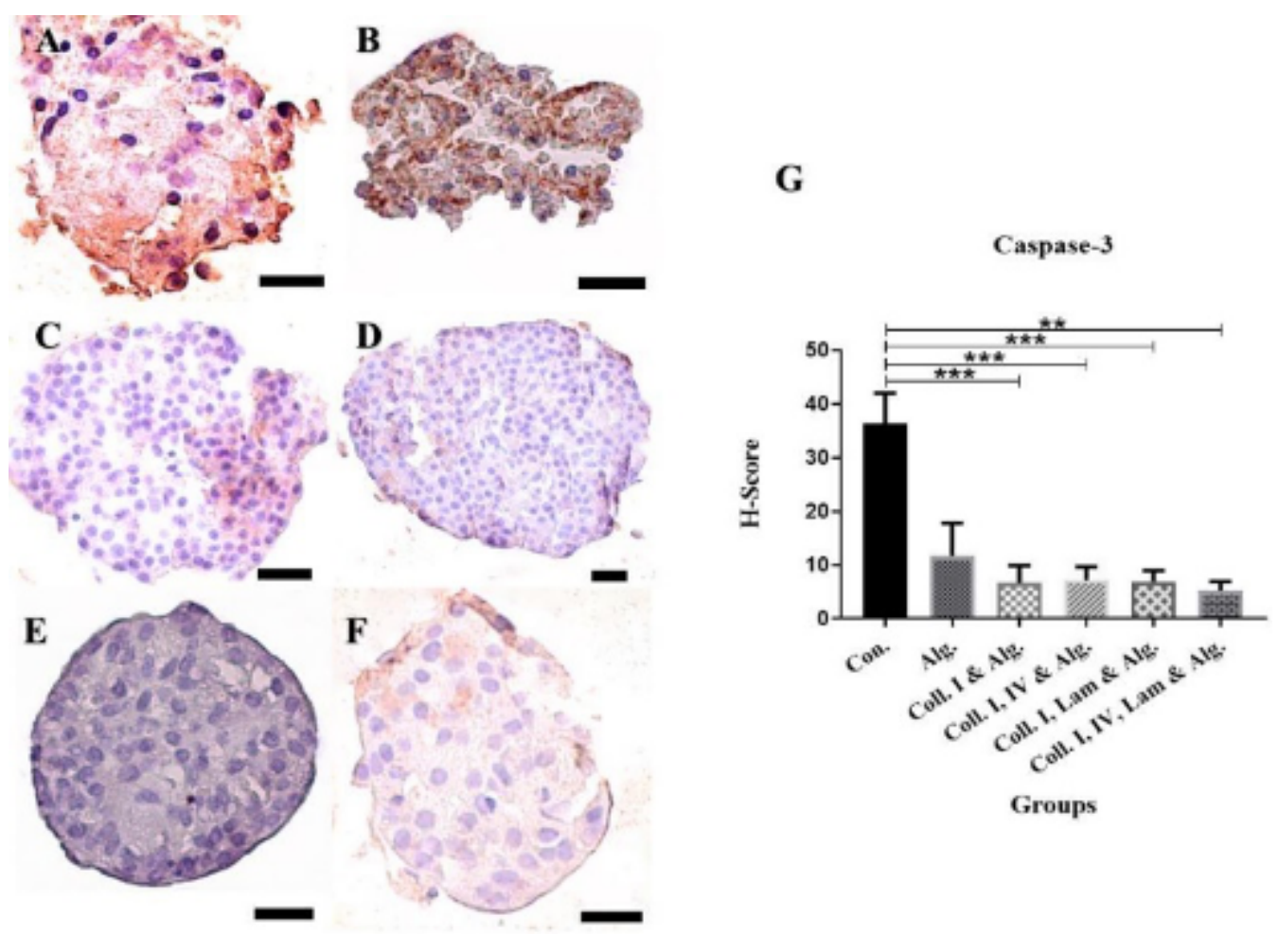

Figure 8

Immunocytochemistry analysis of active caspase-3 protein expression in different groups. The brown spots are the positive areas and the nuclei are visible in blue (A-F). The histogram shows the H-score in each group (G). Data are reported as Mean \pm SEM. (Measurement index of images $=20 \mu \mathrm{m}$. $*$ : $P>0.01$ and ***: $\mathrm{P}<0.001) \mathrm{A}$ : Control group (Con.), B: Alginate hydrogel group (Alg.), C: Collagen I microspheres incorporated in alginate hydrogel group (Coll. I \& Alg.), D: Collagen I microspheres containing collagen IV incorporated in alginate hydrogel group (Coll. I, IV \& Alg.), E: Collagen I microspheres containing laminin incorporated in alginate hydrogel group (Coll. I, Lam \& Alg.), and F: Collagen I microspheres containing collagen IV and laminin incorporated in alginate hydrogel group (Coll. I, IV, Lam \& Alg.). 

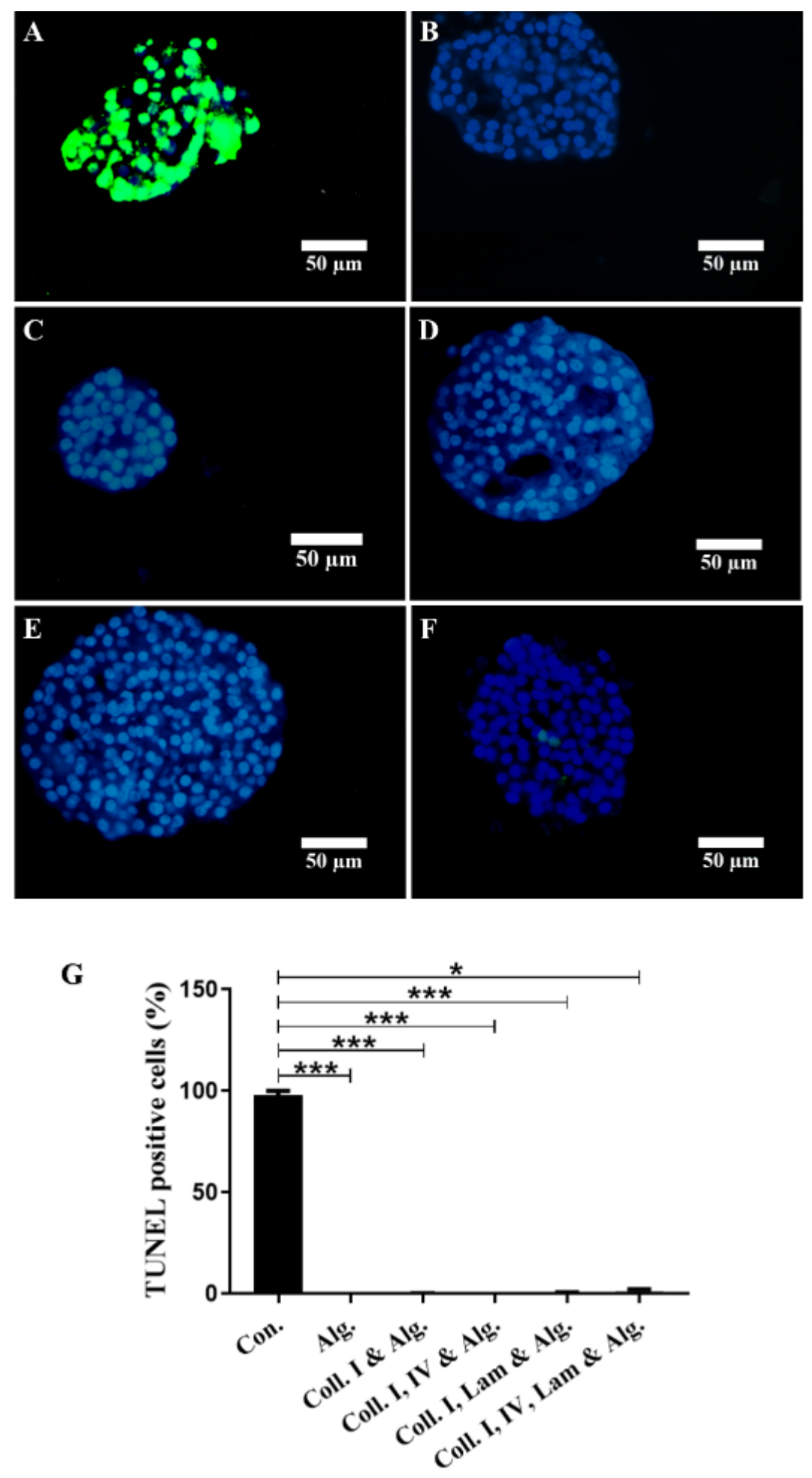

Groups

\section{Figure 9}

TUNEL staining in different groups. The green spots as the TUNEL positive cells and the nuclei are visible in blue (A-F). The histogram shows the percentage of TUNEL positive cells in each group (G). Data are reported as Mean \pm SEM. (*: $P<0.05$ and $* \star *: P<0.001)$ A: Control group (Con.), B: Alginate hydrogel group (Alg.), C: Collagen I microspheres incorporated in alginate hydrogel group (Coll. I \& Alg.), D: Collagen I microspheres containing collagen IV incorporated in alginate hydrogel group (Coll. I, IV \& Alg.), E: 
Collagen I microspheres containing laminin incorporated in alginate hydrogel group (Coll. I, Lam \& Alg.), and F: Collagen I microspheres containing collagen IV and laminin incorporated in alginate hydrogel group (Coll. I, IV, Lam \& Alg.).

A

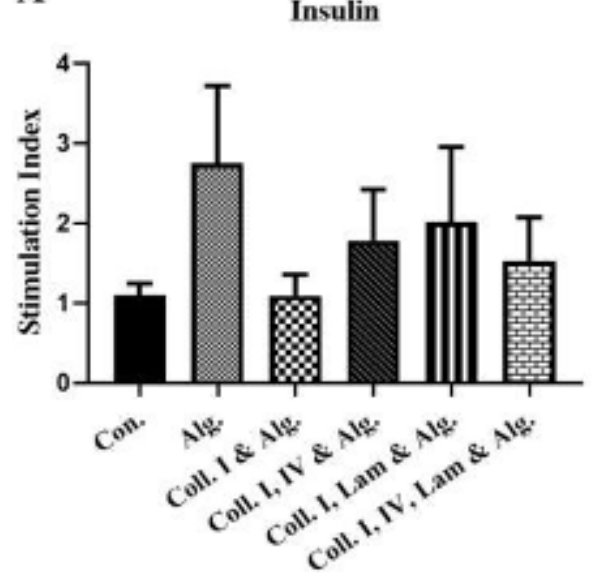

Groups

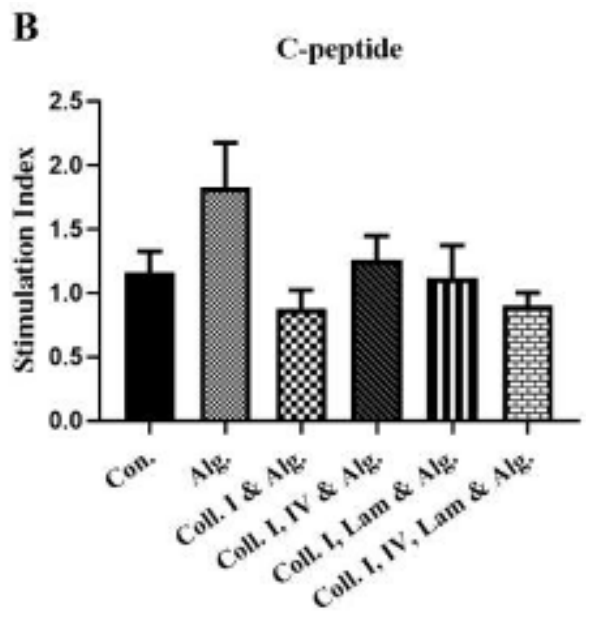

Groups
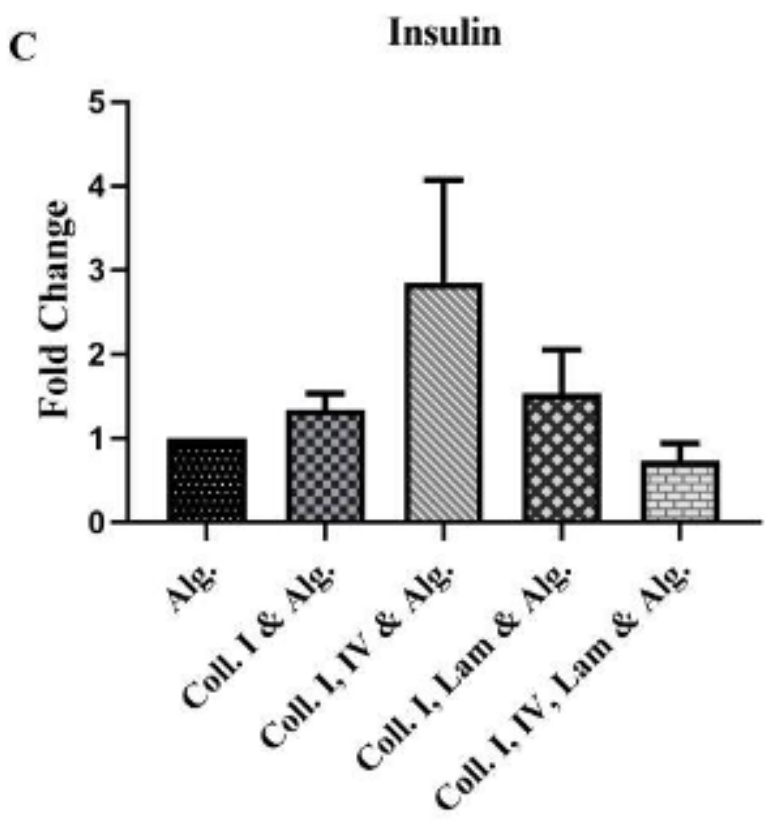

Groups

\section{Figure 10}

The evaluation of insulin (A) and C-peptide (B) stimulation indexes in the presence and absence of hydrogel scaffolds. Data are reported as Mean \pm SEM. (Alg.): Alginate hydrogel group, (Coll. I \& Alg.): Collagen I microspheres incorporated in alginate hydrogel group, (Coll. I, IV \& Alg.): Collagen I microspheres containing collagen IV incorporated in alginate hydrogel group, (Coll. I, Lam \& Alg.): Collagen I microspheres containing laminin incorporated in alginate hydrogel group, and (Coll. I, IV, Lam \& Alg.): Collagen I microspheres containing collagen IV and laminin incorporated in alginate hydrogel group. 


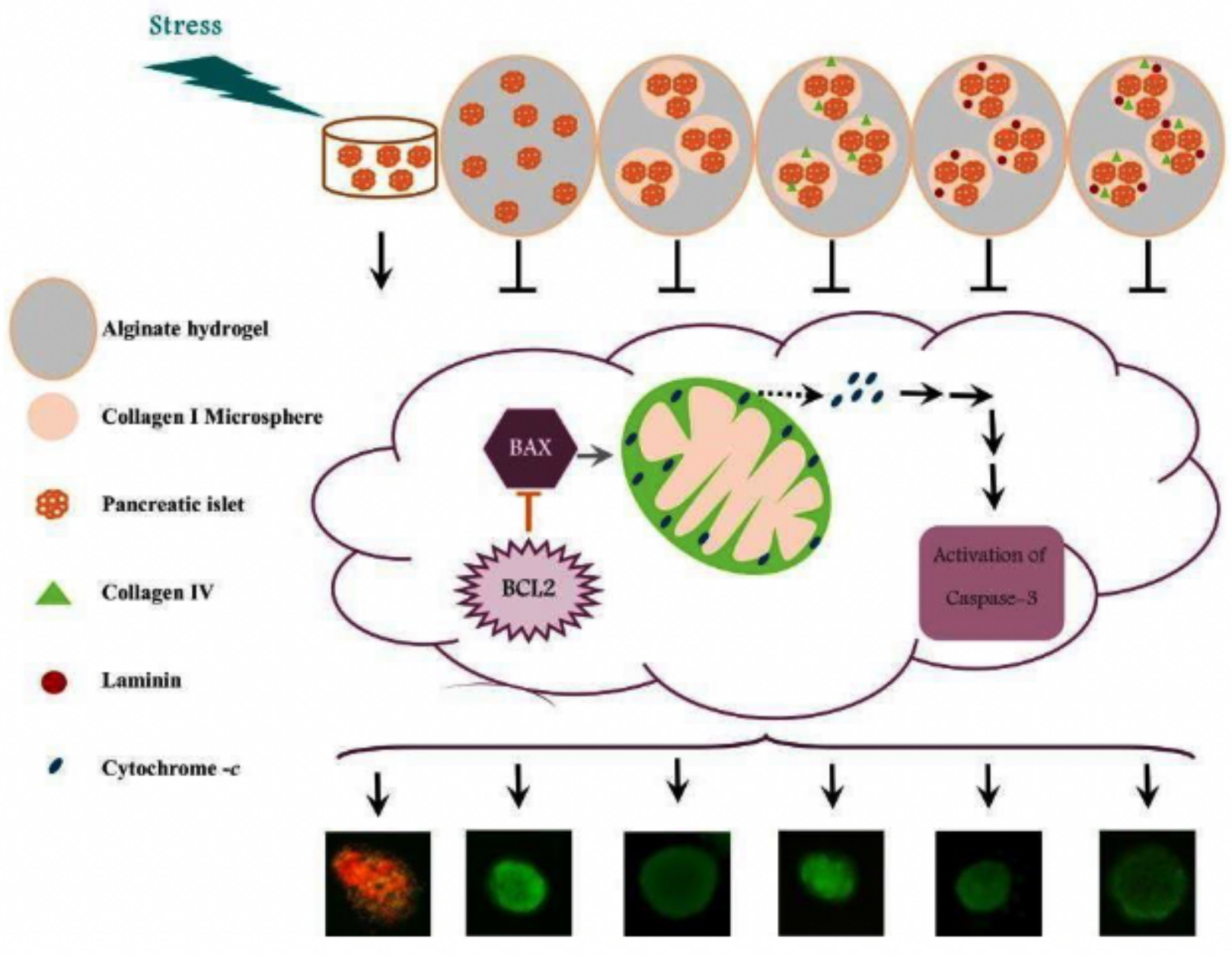

Figure 11

A schematic illustration of hydrogel scaffolds effect on islets apoptosis and viability. The enclosed area represents the internal pathway of apoptosis. 